\title{
A Validation on COVID19 Community Spreading: Probabilistic Approach
}

JAYDIP DATTA ( $\sim$ deepjoy1972@gmail.com )

Indian Institute of Chemical Engineers (CRC) https://orcid.org/0000-0002-0013-5269

\section{Method Article}

Keywords: Theory of Probability , Composite Probability , Viral load, Density function , nth Degree polynomial equation ,Community Spreading , Bioenergetics / Randomness, Mortality rate ,Taylor's expansion series , G-type Variant, S -type variant, Rational Fit, Harris Fit

Posted Date: February 3rd, 2021

DOl: https://doi.org/10.21203/rs.3.rs-99507/v2

License: (1) This work is licensed under a Creative Commons Attribution 4.0 International License. Read Full License 


\section{Abstract}

The objective of this study $(1,2)$ is to validate the viral load alternatively in terms of density function(3). The re-combination of specific mutants leading to spreading of infection denser. The denser is the viral load $(1,2)$ the infectivity will be more probable or susceptible. This viral density ultimately tends to community spreading of that specific mutants and proportional to mortality rate (6). Finally the probability density $(3,5)$ is validated to quantify - a chaotic spreading of Covid19 Virus ( 9 ) leads to community spreading ( 10 ).The community spreading ( 11 ) by different new spike mutants like D614G( 12 ), Del69-70 (13,14, ) are also considered as specific cases of probabilities . The G-type variant depends on age and S-type variant depends on different regions within a community $(12,13,14)$.

\section{Method}

To explain this spreading divergence we have to apply the theory of Probability $(P)$ of an event as Mathematical probability ie $=(p / p+q)$ where $p=$ number of ways that the event can occur,$q=$ number of ways of non-occurrence of the event . Let us consider a simple event is a composite one. The probability of a composite event as a product of the individual simple probabilities ie $P(A)$ So the event building composite probabibility ( 4 ) will be equal to $\mathrm{P}(\mathrm{A}){ }^{\wedge} 2$

$=(p / p+q)^{\wedge} 2$ _equation $(1)$.

Now considering composite Probability as Union of two individual probabilities we get:

$P(A) U P(A)=P(A)$ Union $P(A)=P(A)^{\wedge} 2=[P(A)]^{\wedge} 2 U[P(A) \wedge 2]=P(A)^{\wedge} 4=\ldots \ldots .$.

$\ldots . .\left[P(A)^{\wedge} n\right] \_$equation $(2)[n=$ Integer $=0,1,2 \ldots \ldots \ldots \ldots .$.$] .$

This is known as divergence theorem of Probability.

From the theorem [equation ( 2 ) so data can be generated by the following equation derived from probability statistics.

Viral load or density function becomes $(y)=[P(A)]^{\wedge} n=1+P(A)+P(A) \wedge 2+$ $P(A)^{\wedge} n$ equation ( 3 )

Taking differential operator $(D=d / d x)$ on $y=D(y)=f^{\prime}(y)=n^{\star}[P(A)]^{\wedge}(n-1)$

$f^{\prime \prime}(y)=n(n-1) *[P(A)]^{\wedge}(n-2), f^{\prime \prime}(y)=n(n-1)(n-2)\left[P(A)^{\wedge}(n-3)\right.$

Using Taylor's expansion ( 7 ) series $\Sigma D n(y)=f^{\prime}(y)+f^{\prime \prime}(y)+f^{\prime \prime \prime}(y)+$ Infinity equation (4)

Finally validating the probability density in terms of bio energetic probability ( 9 ) by Nernst's 
Theorem as $\Delta S=$ Change in entropy $=\mathrm{K} \operatorname{Ln}\left[\mathrm{P}(\mathrm{A})^{\wedge} \mathrm{n}\right]$, Where $\mathrm{K}=$ Boltzmann Constant

equation ( 5 ), the randomness of spreading of infection. This randomness

can be also explained by the bio energetic state function Entropy ( 9 ).

The spreading of G-type mutant ( D614G) , S-type mutant ( Del 69-70) can be validated as follows :

D614G validation ( 12 ) - This type of infection is more fatal starting from 46 Yrs above statistically validated by Morgan -Morgan -Finney (MMF) regression.

(Del 69-70) validation (13,14)- This type of new type of infection with deleted residues of amino acid 69-70 could also be investigated W.E.F last year of October ,2020 in UK or around London city also termed as Variant of Investigation( VUI ). The scattered data in and around this community also validated by Rational and Harris fits of probabilistic regression.

\section{Results}

The results may be considered by the availability of data by using the nth degree polynomial equation ( 3,4$)$. The real epidemiological parameter is mortality rate [ equation $(1), 6$ ] is defined as the ratio of the population affected by the infection to the total population of a specific community per unit time. The more the value of $y$ the denser will be viral load or probability (3) the higher will be mortality rate (5). The equation ( 5 ), 9 represents the chaos of viral infectivity more virulent more probable. Community spreading is nothing but the probabilistic spreading of infection density in a particular community .The theoretically calculated viral load [equation (5),9] can be better realised by above two variants ( $G$ and $S$ ) in various regions of Europe where spike

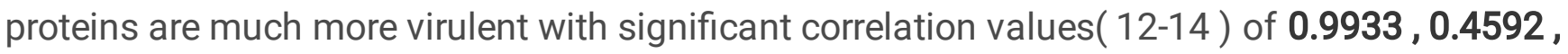

0.3594 p value $<0.01$ tolerance, Confidence Interval $(\mathrm{Cl})>95 \%$ ie more probable for community spreading .

\section{Conclusion}

The Covid - Infection density is hypothesised to divergence probability $(3,5)$ to

find out the density function ( 3 ) of mortality rate (6).The thermodynamic state function entropy 
( $\Delta \mathrm{S}$ ) can be correlated to viral density or chaos or more virulent ( 9 ) ultimately leading to validate community spreading ( 10 ). The theoretical model equation ( 5 ) ,9 can be extended to MMF -

Rational - Harris model fits $(12,13)$ thus validate the community spreading of different newer mutating SARS-COV-2 strains like D614G, VUI (Del69-70) according to age(12 ), number of days or regional communities ( 14$)$.

\section{Declarations}

I hereby declare that I have no potential conflict of interest/ Competing interest.

\section{References}

1. JAYDIP DATTA . COVID19 - A Correlation Study of Infection Fatality Rate vs Age, 20 October 2020, PREPRINT (Version 2) available at Research Square [+https://doi.org/10.21203/rs.3.rs-85482/v2+]

2. Jaydip Datta. Mathematical and Statistical Study on COVID19- SIR Model, 12 October 2020, PREPRINT (Version 2) available at Research Square [+https://doi.org/10.21203/rs.3.rs-89766/v2+]

3. Jaydip Datta , https://ideas.repec.org/p/osf/osfxxx/3f9ct.html , 2020

4. C.RAKHSIT , Physical Chemistry, Seventh Edition , 2004 , P. 28

5. https://en.wikipedia.org/wiki/Divergence_(statistics)

6. https://en.wikipedia.org/wiki/Mortality_rate

7. https://www.mathsisfun.com/algebra/taylor-series.html

8. Figure $-I$, (magoosh.com )

9. C.RAKHSIT, Physical Chemistry, Seventh Edition , 2004 , P. 253.

10. A great realisation to Probability statistics and pandemics, Carlo Rovelli -https://nature.us17.listmanage.com/track/click?u=2c6057c528fdc6f73fa196d9d\&id=d794fdc7b9\&e=5944515236

11. JAYDIP DATTA . A Validation on COVID19 Community Spreading: Probabilistic Approach\&nbsp;\&nbsp; 30 October 2020, PREPRINT (Version 1) available at Research Square [https://doi.org/10.21203/rs.3.rs-99507/v1]

12. JAYDIP DATTA . Growth of D614G Virus : Morgan - Morgan - Finney\&nbsp;( MMF ) Regression\&nbsp; 15 October 2020, PREPRINT (Version 1) available at Research Square [https://doi.org/10.21203/rs.3.rs-92776/v1] .

13. JAYDIP DATTA. (2021). DATA ANALYSIS - SARS-COV-2 ( Del69-70 VARIANT ) - NEW UK MUTANTS (Version Version 1.0) [Data set]. Zenodo. http://doi.org/10.5281/zenodo.4492328 .

14. https://www.academia.edu/45033874/SARS_COV_2_Del69_70_VARIANT_NEW_UK_MUTANTS , JAYDIP DATTA . 
Figures

$$
\begin{aligned}
& \sum_{k=0}^{n} \frac{f^{(k)}(c)}{k !}(x-c)^{k} \\
& =f(c)+f^{\prime}(c)(x-c)+\frac{f^{\prime \prime}(c)}{2}(x-c)^{2} \\
& \quad+\frac{f^{\prime \prime \prime}(c)}{6}(x-c)^{3}+\cdots+\frac{f^{(n)}(c)}{n !}(x-c)^{n}
\end{aligned}
$$

Figure 1

Taylor's Polynomial Series 


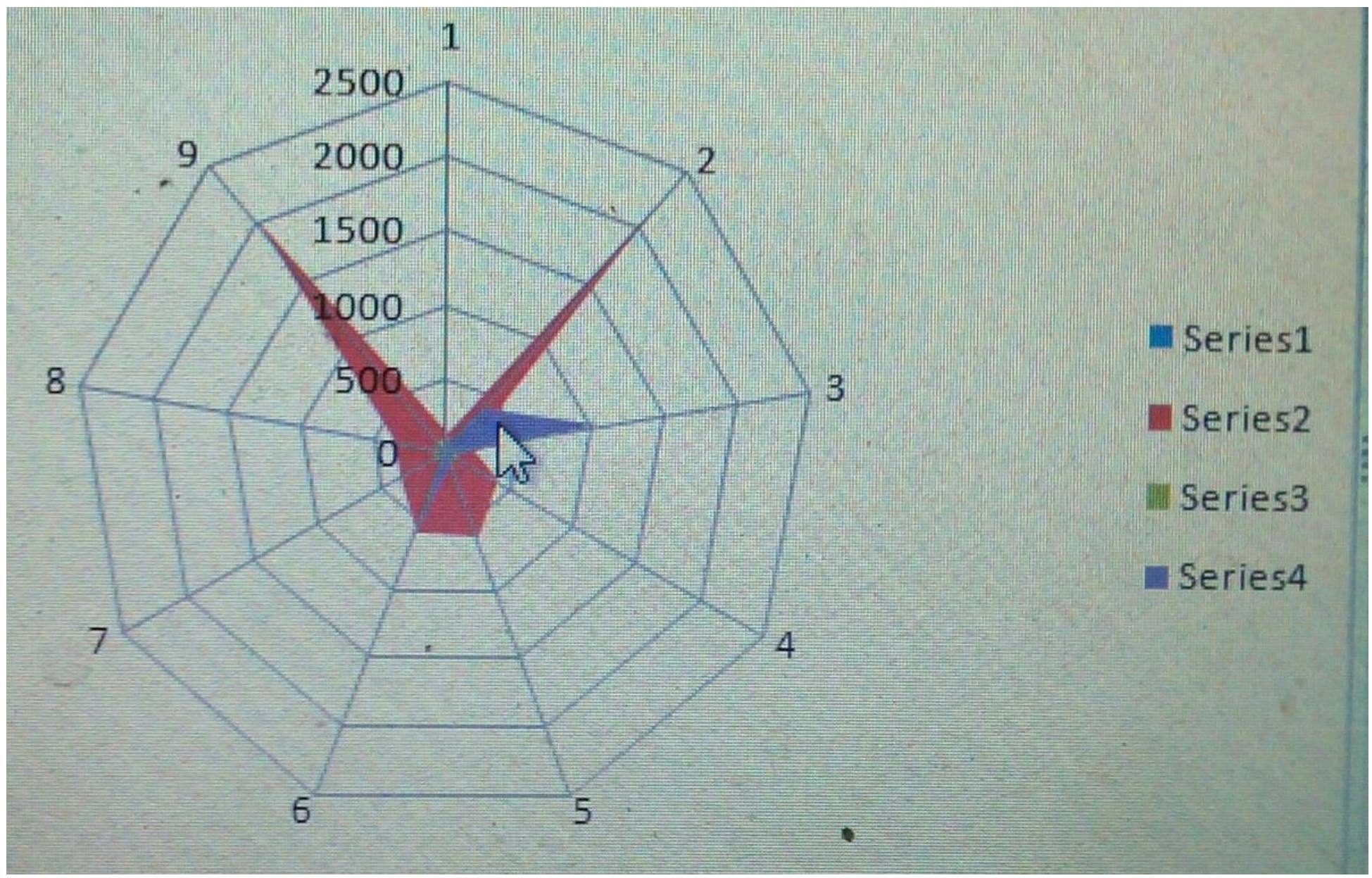

Figure 2

VUI ( Del69-70)

\section{Supplementary Files}

This is a list of supplementary files associated with this preprint. Click to download.

- 6ca2ea2f9e00cd2f44a0b9e7.docx 